\title{
Evaluation of Serum Ceruloplasmin Levels as a Biomarker for Oxidative Stress in Patients With Diabetic Retinopathy
}

Gurunadh Satyanarayana ${ }^{1}$, Narendra Keisham ${ }^{2}$, Hitender S. Batra ${ }^{3}$, Subrahmanya Murti V ${ }^{4}$, Mansur Khan ${ }^{5}$, Sandeep Gupta ${ }^{6}$, Vikram Mahindra ${ }^{7}$

1. Ophthalmology, GSL Medical College, Rajahmundry, IND 2. Ophthalmology, Military Hospital, Jabalpur, IND 3. Biochemistry, Military Hospital, Kirkee, IND 4. Cardiology, Smt. Nathiba Hargovandas Lakhmichand (NHL) Municipal Medical College, Ahmedabad, IND 5. Vitreo-Retina, Command Hospital Air Force, Bangalore, IND 6. Cornea, Command Hospital Chandimandir, Panchkula, IND 7. Ophthalmology, Armed Forces Medical Services, Pune, IND

Corresponding author: Gurunadh Satyanarayana, gurunadhsatyanarayana@gmail.com

\section{Abstract}

\section{Background}

Elevated serum ceruloplasmin is a biomarker for oxidative stress. Diabetes mellitus (DM) is known to be a state of oxidative stress which causes complications of DM including diabetic retinopathy (DR). The role of ceruloplasmin in DR is still unclear.

\section{Methods}

Ninety patients of DM were included as cases and after evaluation sub-grouped as those with no DR, nonproliferative DR (NPDR) and proliferative DR (PDR). Serum ceruloplasmin levels were tested in all cases as well as in equal numbers of age and sex-matched controls without DM. Statistical analysis was done with $\mathrm{p}<0.05$ taken as significant.

\section{Results}

Serum ceruloplasmin was significantly higher among cases as compared to controls $(1222.82 \pm 306.15 \mathrm{IU} / \mathrm{L}$ versus $868.38 \pm 198.80 \mathrm{IU} / \mathrm{L}, \mathrm{p}<0.01$ ). There was no statistical difference between serum ceruloplasmin values in No DR, NPDR and PDR. On receiver operator characteristic curve (ROC) analysis for serum ceruloplasmin as a test for discriminating various parameters, it was seen that serum ceruloplasmin was a good test for discriminating DM from no DM (area under receiver operator characteristic $\{$ AUROC $\}=0.814,95 \% \mathrm{CI}=0.749$ $0.868, \mathrm{p}<0.0001$ ) with a cut point of $>1093 \mathrm{IU} / \mathrm{L}$ yielding a sensitivity of $63.33 \%$ and specificity of $87.78 \%$. Ceruloplasmin as a test was not found to significantly discriminate DR (total) from no DR, NPDR from no DR, PDR from no DR and PDR from NPDR.

Review began 01/18/2021 Review ended 01/29/2021 Published 02/02/2021

\section{(c) Copyright 2021}

Satyanarayana et al. This is an open access article distributed under the terms of the Creative Commons Attribution License CC-BY 4.0., which permits unrestricted use, distribution, and reproduction in any medium, provided the original author and source are credited.

\section{Conclusion}

Serum ceruloplasmin levels are significantly raised in patients with DM. However, serum ceruloplasmin levels do not correlate with DR severity.

Categories: Ophthalmology

Keywords: diabetes mellitus, diabetic retinopathy, ceruloplasmin, oxidative stress

\section{Introduction}

Diabetic retinopathy (DR) is a leading cause of blindness in the world. As per data from the nationwide Indian Council of Medical Research (ICMR) - InDIAB study, 7.3\% of Indians suffer from diabetes mellitus (DM) which translates to a huge burden of around 65 million people with diabetes and 77.2 million people with prediabetes [1]. Further, by 2030, India is projected to become the country with the highest burden of diabetes with about 80 million cases. As the global prevalence of diabetes increases, so will the number of people with diabetes-related complications including DR. As per a 2016 study, there are about 22 million patients with diabetic retinopathy in India [2].

Every patient with DM has the potential to develop DR over a period of time with the frequency and severity of DR being related to the duration of diabetes. Therefore, early detection of DR and its management is important to prevent disabling visual loss from DR. Several theories exist on the mechanism of diabetic complications. It has been postulated that DM is a state of chronic inflammation with increased oxidative stress. Glucose is usually metabolized by the glycolytic and tricarboxylic acid cycle pathways yielding reducing equivalents which drive the synthesis of adenosine triphosphate (ATP) by oxidative phosphorylation. However, oxidative phosphorylation also produces free radicals or reactive oxygen species (ROS), such as superoxide anion, whose production is increased by high levels of glucose [3]. 
Elevated levels of ROS reduce levels of nitric oxide which promotes endothelial activation, consequent leucocyte adhesion and reduced barrier function. All these changes cause microangiopathy which causes the changes observed in DR [4]. Deranged homeostasis of ROS has been implicated in the development of DR $[5,6]$. In this setting, various methods to quantify oxidative stress have been developed. One of the known markers of oxidative stress is ceruloplasmin. Ceruloplasmin is a circulating blue multi-copper oxidase that contains $>95 \%$ of copper in the plasma. Ceruloplasmin exhibits a copper-dependent oxidase activity, which is associated with oxidation of Fe2+ (ferrous iron) into Fe3+ (ferric iron). An increase in serum ceruloplasmin levels has been reported in type 2 DM [7]. The primary physiologic role of ceruloplasmin involves plasma redox reactions. Ceruloplasmin permits the incorporation of iron into transferrin without the formation of toxic iron products. Oxygen is directly reduced to water in that redox reaction and may also represent the mechanism by which ceruloplasmin inhibits superoxide induced lipid peroxidation.

Under physiologic conditions, ceruloplasmin is also important in the control of membrane lipid oxidation, probably by direct oxidation of cations, thus preventing their catalysis of lipid peroxidation [8]. Increased ceruloplasmin levels seen in diabetes mellitus may be a protective response to an increase in circulating unbound $\mathrm{Fe} 2+$, which would act as a catalyst for further free radical-induced lipoperoxidation [9]. Therefore, elevated plasma ceruloplasmin levels could signal abnormally high oxidant stress. A study by Inoue et al. found that ceruloplasmin was a marker of oxidative stress in DM [10]. Therefore, this study is aimed at finding out whether serum ceruloplasmin is higher in patients of DM (with and without DR) compared to controls without DM and also whether levels of serum ceruloplasmin have any relation with the severity of DR. This may help to serve as a marker of uncontrolled DM and DR, which may help in improving their management and patient outcomes.

\section{Materials And Methods}

A prospective, observational, hospital-based, case-control study was conducted on 90 patients of type 2 DM attending eye OPD at a tertiary care hospital in western India. Another 90 age- and sex-matched individuals without DM served as controls.

A complete medical history was taken for all the cases and control subjects with an emphasis on the points that may be confounders for levels of serum ceruloplasmin. These were diabetes mellitus, hypertension, renal disease, pregnancy, oral contraceptive pills, copper toxicity, zinc deficiency, rheumatoid arthritis or any acute or chronic inflammatory disease.

All patients underwent an ophthalmic examination and investigations. These investigations were bestcorrected visual acuity by Snellen's visual acuity chart, slit-lamp biomicroscopic examination of the cornea, anterior chamber, lens and anterior vitreous. Dilated fundus examination was done with direct ophthalmoscopy, slit-lamp bio-microscopy with $90 \mathrm{D}$ lens and indirect ophthalmoscopy where required. Intraocular pressure was estimated by Goldman applanation tonometry. Systemic examination was conducted including blood pressure measurement using an electronic manometer. Optical coherence tomography (OCT) and fundus fluorescein angiography (FFA) was done in all cases of DR. The grading of DR was based on Early Treatment Diabetic Retinopathy Study (ETDRS) classification. Laboratory investigations consisted of serum ceruloplasmin level, fasting (FBS) and post-prandial blood sugar (PPBS) levels, glycosylated hemoglobin levels (HbA1c) and serum urea and creatinine levels.

Based on the clinical picture and corroborated by FFA patients with DR were grouped as Groups A, B, C and D. Group A consisted of 30 diabetics with no DR (No DR). Group B consisted of 30 diabetics with mild and moderate non-proliferative diabetic retinopathy (NPDR). Group C consisted of 30 diabetics with severe/very severe NPDR and proliferative diabetic retinopathy (PDR). These two states of DR were merged because of similar management strategies. Groups A, B and C together form the study group of 90 patients. Group D consisted of 90 age- and sex-matched controls.

Patients of type 2 diabetes mellitus attending Eye OPD were included in the study. Patients unwilling to give consent were excluded along with patients with coexisting diabetic nephropathy, patients with Wilson's/ Menke's Disease, patients with any other systemic acute or chronic inflammatory disease, patients having retinopathy other than $\mathrm{DR}$,

Serum ceruloplasmin measurement was done by the Somani and Ambade method. Ceruloplasmin is a copper-containing alpha 2 glycoprotein which possesses significant oxidase activity against ferrous ions. Chromogen $(0.5 \mathrm{mmol} / \mathrm{L})$ solution (Reagent-1): was made by dissolving $159.65 \mathrm{mg}$ of norfloxacin in $1000 \mathrm{~mL}$ of acetate buffer $(0.45 \mathrm{~mol} / \mathrm{L}, \mathrm{pH} 5.4)$ containing $0.2 \%$ Triton X-100. Substrate $(2.04 \mathrm{mmol} / \mathrm{L})$ solution (Reagent-2): was made by sequentially dissolving, $320 \mathrm{mg}$ of dithiothreitol (DTT) and $800 \mathrm{mg}$ of ferrous ammonium sulfate, $\mathrm{Fe}(\mathrm{NH} 4) 2(\mathrm{SO} 4) 2 \cdot 6 \mathrm{H} 2 \mathrm{O}$, in $1000 \mathrm{~mL}$ of distilled water. Both Reagent-1 and Reagent-2 were stable for more than 6 months at $4{ }^{\circ} \mathrm{C}$ as well as at room temperature. Standard $(6.0 \mathrm{mmol} / \mathrm{L})$ : was made by dissolving $2.896 \mathrm{~g}$ of ammonium iron (III) sulfate dodecahydrate, $\mathrm{NH} 4 \mathrm{Fe}(\mathrm{SO} 4) 2 \cdot 12 \mathrm{H} 2 \mathrm{O}$, in $1000 \mathrm{~mL}$ of acetic acid $(0.20 \mathrm{mmol} / \mathrm{L})$. Serum sample $(50 \mu \mathrm{L})$ was added to $1000 \mu \mathrm{L}$ of Reagent-1 and mixed. After a 1min interval, $150 \mu \mathrm{L}$ of Reagent- 2 was added. The mixture was aspirated into Shimadzu CL-750 spectrophotometer with the following settings: measuring wavelength $377 \mathrm{~nm}$, lag period $10 \mathrm{~s}$, kinetics time $30 \mathrm{~s}$, temperature $37^{\circ} \mathrm{C}$, aspiration volume $1 \mathrm{~mL}$ and factor 2012 . For the blank correction, a blank was run 


\section{Cureus}

using distilled water in place of the serum sample.

\section{Statistical analysis}

There were a total of 90 subjects and 90 controls. For comparison of means between groups, unpaired t-tests and analysis of variance (ANOVA) were used. IBM Statistical Package for the Social Sciences (SPSS) Package 16.0 (IBM, Armonk, NY) was used for analysis.

\section{Results}

The mean age of the cases was $59.74 \pm 6.77$ years which was well matched to controls. As expected, the mean fasting blood sugar (FBS), post-prandial blood sugar (PPBS) and HbA1C were significantly higher in cases as compared to controls. There was no statistical difference in serum values of urea and creatinine among cases and controls. Serum ceruloplasmin was significantly higher among cases (with DM) as compared to controls $(1222.82 \pm 306.15 \mathrm{IU} / \mathrm{L}$ versus $868.38 \pm 198.80 \mathrm{IU} / \mathrm{L}, \mathrm{p}<0.01)$ (Table 1$)$.

\begin{tabular}{|c|c|c|c|}
\hline Parameter & Cases (patients with DM) & Controls (without DM) & P-value \\
\hline Age (years) & $59.74 \pm 6.77$ & $58.22 \pm 7.95$ & 0.129 \\
\hline FBS (mg/dL) & $129.14 \pm 15.65$ & $84.93 \pm 9.80$ & $<0.001$ \\
\hline PPBS (mg/dL) & $219.70 \pm 34.35$ & $128.26 \pm 15.71$ & $<0.001$ \\
\hline Serum Urea (mg/dL) & $25.93 \pm 6.77$ & $23.62 \pm 6.22$ & 0.016 \\
\hline Serum Creatinine (mg/dL) & $0.87 \pm 0.16$ & $0.89 \pm 0.17$ & 0.412 \\
\hline Serum Cerulopasmin (IU/L) & $1222.82 \pm 306.15$ & $868.38 \pm 198.80$ & $<0.01$ \\
\hline HbA1C & $8.84 \pm 1.28$ & $5.94 \pm 0.93$ & $<0.01$ \\
\hline
\end{tabular}

\section{TABLE 1: Comparison of cases and controls}

DM: diabetes mellitus; HbA1C: glycosylated hemoglobin; FBS: fasting blood sugar; PPBS: post-prandial blood sugar

Of a total 90 cases of DM, subdivision was done as those without DR (no DR $=30$ patients), with nonproliferative DR (NPDR = 30 patients) and those with proliferative $\mathrm{DR}$ ( $\mathrm{PDR}=30$ patients). Comparisons among these subgroups are shown in Table 2. HbA1C was significantly lower in NPDR as compared to No DR $(8.65 \pm 1.28$ versus $9.41 \pm 1.11, \mathrm{p}<0.05)$ and in PDR as compared to No DR $(8.45 \pm 1.28$ versus $9.41 \pm 1.11$, $\mathrm{p}<0.05)$. There was no statistical difference between HbA1C values in PDR and NPDR. Further, there was no statistical difference between serum ceruloplasmin values in No DR, NPDR and PDR (Table 2). 


\section{Cureus}

\begin{tabular}{|c|c|c|c|c|c|c|}
\hline Parameter & $\begin{array}{l}\text { Diabetes mellitus without } \\
\text { retinopathy (No DR) }\end{array}$ & NPDR & PDR & $\begin{array}{l}\text { P-value (no DR } \\
\text { versus NPDR) }\end{array}$ & $\begin{array}{l}\text { P-value (no DR } \\
\text { versus PDR) }\end{array}$ & $\begin{array}{l}\text { P-value (NPDR } \\
\text { versus PDR) }\end{array}$ \\
\hline Age (years) & $58.77 \pm 8.15$ & $\begin{array}{l}57.87 \pm \\
5.37\end{array}$ & $\begin{array}{l}62.60 \pm \\
5.67\end{array}$ & 0.594 & 0.025 & 0.006 \\
\hline FBS & $123.37 \pm 9.53$ & $\begin{array}{l}128.40 \pm \\
10.42\end{array}$ & $\begin{array}{l}135.67 \pm \\
21.76\end{array}$ & 0.196 & 0.002 & 0.064 \\
\hline PPBS & $209.83 \pm 23.34$ & $\begin{array}{l}219.93 \pm \\
25.05\end{array}$ & $\begin{array}{l}229.33 \pm \\
47.45\end{array}$ & 0.250 & 0.028 & 0.284 \\
\hline $\begin{array}{l}\text { Serum Urea } \\
(\mathrm{mg} / \mathrm{dL})\end{array}$ & $23.70 \pm 5.83$ & $\begin{array}{l}25.93 \pm \\
5.99\end{array}$ & $\begin{array}{l}28.17 \pm \\
7.77\end{array}$ & 0.193 & 0.010 & 0.193 \\
\hline $\begin{array}{l}\text { Serum } \\
\text { Creatinine } \\
(\mathrm{mg} / \mathrm{dL})\end{array}$ & $0.84 \pm 0.17$ & $\begin{array}{l}0.83 \pm \\
0.16\end{array}$ & $\begin{array}{l}0.93 \pm \\
0.13\end{array}$ & 0.740 & 0.022 & 0.009 \\
\hline $\begin{array}{l}\text { Serum } \\
\text { Ceruloplasmin } \\
\text { (IU/L) }\end{array}$ & $1167.60 \pm 295.69$ & $\begin{array}{l}1238.33 \pm \\
287.40\end{array}$ & $\begin{array}{l}1262.53 \pm \\
335.53\end{array}$ & 0.375 & 0.234 & 0.761 \\
\hline HbA1C & $9.41 \pm 1.11$ & $\begin{array}{l}8.65 \pm \\
1.28\end{array}$ & $\begin{array}{l}8.45 \pm \\
1.28\end{array}$ & 0.018 & 0.003 & 0.529 \\
\hline
\end{tabular}

\section{TABLE 2: Distribution of cases with comparisons among the subgroups}

NPDR: non-proliferative diabetic retinopathy; PDR: proliferative diabetic retinopathy; FBS: fasting blood sugar; PPBS: post-prandial blood sugar; HbA1C: glycosylated hemoglobin

On receiver operator characteristic (ROC) curve analysis for serum ceruloplasmin as a test for discriminating various parameters, it was seen that serum ceruloplasmin was a good test for discriminating DM from no DM (area under ROC curve $=0.814,95 \% \mathrm{CI}=0.749-0.868, \mathrm{p}<0.0001$ ) with a cut point of $>1093 \mathrm{IU} / \mathrm{L}$ yielding a sensitivity of $63.33 \%$ and specificity of $87.78 \%$. Ceruloplasmin as a test was not found to significantly discriminate DR (total) from no DR, NPDR from no DR, PDR from no DR and PDR from NPDR (Figure 1).
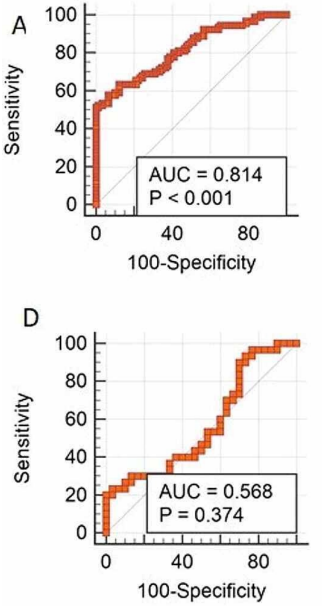

B
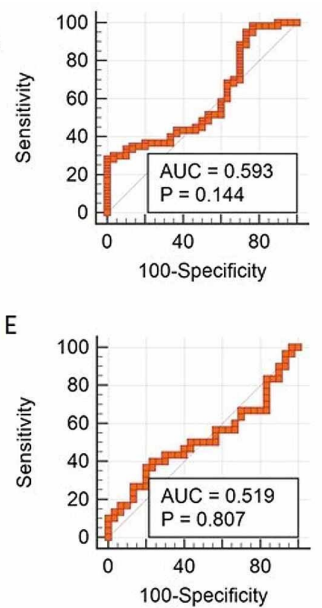

C

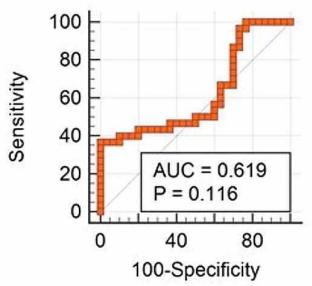

FIGURE 1: Area under receiver operator characteristic (AUROC) curves for ceruloplasmin

(A) DM versus No DM; (B) DR versus No DR; (C) PDR versus No DR; (D) NPDR versus No DR; (E) PDR versus NPDR

DM: diabetes mellitus; DR: diabetic retinopathy; PDR: proliferative diabetic retinopathy; NPDR: nonproliferative diabetic retinopathy 


\section{Discussion}

In the present study, serum ceruloplasmin was significantly higher in patients with DM (cases) as compared to controls (without DM). Existing literature has also shown higher levels of ceruloplasmin in patients with DM. In the largest data on ceruloplasmin in patients with DM from Japan, serum ceruloplasmin was significantly higher in people with DM compared to those without [9]. The same conclusion was also drawn from a recent study from Nepal, which also showed a correlation of higher low-density cholesterol (LDL) to higher ceruloplasmin levels. These findings suggest that DM is a state of increased oxidative stress [11].

In the present study, there was no statistical difference in serum levels of ceruloplasmin among diabetic patients without retinopathy as compared to those with DR. Further, among patients of DR, there was no significant difference in ceruloplasmin levels among patients with proliferative DR versus non-proliferative DR. This suggests that ceruloplasmin levels may not increase with increasing retinopathy and thus may not help to discriminate the severity of retinopathy. The role of serum ceruloplasmin levels in diabetic complications has been investigated earlier with conflicting results. Oxidative stress has been linked to retinopathy in DM with evidence from animal models available [12]. In a study by Mohora et al. on patients with diabetic foot, patients with retinopathy had significantly higher levels of ceruloplasmin compared to those without [13]. The same study also found a similar trend in diabetic nephropathy and neuropathy wherein there was no significant positive trend in serum ceruloplasmin levels with the severity of the diabetic complication. In a study by Nowak et al., 41 patients with type 1 DM were examined. Interestingly, that study showed that ceruloplasmin levels were lower in those with DR than those without [14]. However, continuing the debate on oxidative stress, in contrast to other studies, a Korean study on diabetic nephropathy found that higher ceruloplasmin levels were associated with faster progression of nephropathy [15].

In the present study, serum HbA1C was significantly lower in NPDR as compared to No DR and in PDR as compared to No DR. There was no statistical difference between HbA1C values in PDR and NPDR. These findings are contrary to the general appreciation that diabetic complications are associated with poor glycemic control. Similar results were seen in a large Chinese study wherein there was no significant positive correlation between retinopathy grade and HbA1C [16]. These may be due to two reasons - patients with PDR and NPDR may have a confounding bias of stricter treatment and onset of retinopathy may have other factors rather than being solely dependent on glycemic status alone. One of the important factors is the presence of coexistent nephropathy. Various studies have found that in the presence of nephropathy, $\mathrm{HbA1C}$ had a less accurate correlation with glycemic control [17]. The 'metabolic memory' theory, as described in the results of many pivotal trials shows that early intensive glycemic control reduces the onset of complications of diabetes [18]. This effect may also confound correlations of the glycemic control with the severity of complications in DM.

In the present study, using ROC analysis, it was found that ceruloplasmin as a test was good in discriminating only DM from no DM and was not useful in discriminating DR from no DR. A ceruloplasmin level $>1093$ IU/L yielded a sensitivity of $63.33 \%$ and specificity of $87.78 \%$ for diagnosing DM (area under receiver operator characteristic $\{$ AUROC $\}=0.814, \mathrm{p}<0.0001$ ). Similar results were obtained by Sharma et al. wherein a cutoff of $46.5 \mathrm{mg} / \mathrm{dL}$ of serum ceruloplasmin had a sensitivity of $87.5 \%$ and specificity of $62 \%$ (AUROC $=0.881, \mathrm{p}<0.01$ ) for discriminating those with DM compared to those without [11]. These results, along with those described above suggest that serum ceruloplasmin is definitely elevated in subjects with DM establishing DM as a state of increased oxidative stress. However, the relationship of serum ceruloplasmin with onset, progression and severity of diabetic complications is less well established and requires further evaluation in larger studies.

Whether treatment with antioxidants has a role in DM is a subject of open debate. Antioxidants are of some benefit in experimental animals with diabetic retinopathy. However, this has not translated into clinical trials with some small uncontrolled clinical trials showing no benefit over placebo [3]. Classical clinical trials like the Diabetes Control and Complications Trial (DCCT) drive home the importance of good glycemic control. In fact, some of the diagnostic cut-offs for DM were derived from data on trends of incidence of retinopathy with rising sugar levels in a classical trial by Dorf et al. on the Pima Indian population in the Amazonian areas, an area with a known higher incidence of DM [19]. These emphasize the fact that the onus still remains on good glycemic control which is the cornerstone of the management of DM.

\section{Study limitations}

The major limitation of the present study is that the sample size is small. This makes it difficult to draw definitive conclusions about the role of ceruloplasmin levels in DR.

\section{Conclusions}

This study started with the question of the role of serum ceruloplasmin levels in DM as also in the main ocular complication of DM i.e., DR. This study has shown that serum values of ceruloplasmin are significantly elevated in patients with DM when compared to controls without DM. However, among patients of DM, there was no statistical difference in levels of serum ceruloplasmin among those with DR 
compared to those without DR. Further, among patients with DR, there was no statistical difference in serum ceruloplasmin levels between those with NPDR compared to those with PDR. Higher ceruloplasmin levels in patients with DM as shown by this study, adds to the already existing body of evidence that DM is a state of increased oxidative stress. However, the reasoning that levels of serum ceruloplasmin would rise with increasing severity of retinopathy because of the presumed increase in anti-oxidant activity was not corroborated by this study.

\section{Additional Information \\ Disclosures}

Human subjects: Consent was obtained or waived by all participants in this study. Animal subjects: All authors have confirmed that this study did not involve animal subjects or tissue. Conflicts of interest: In compliance with the ICMJE uniform disclosure form, all authors declare the following: Payment/services info: All authors have declared that no financial support was received from any organization for the submitted work. Financial relationships: All authors have declared that they have no financial relationships at present or within the previous three years with any organizations that might have an interest in the submitted work. Other relationships: All authors have declared that there are no other relationships or activities that could appear to have influenced the submitted work.

\section{References}

1. Anjana RM, Deepa M, Pradeepa R, et al.: Prevalence of diabetes and prediabetes in 15 states of India: results from the ICMR-INDIAB population-based cross-sectional study. Lancet Diabetes Endocrinol. 2017, 5:585596. 10.1016/s2213-8587(17)30174-2

2. Raman R, Gella L, Srinivasan S, Sharma T: Diabetic retinopathy: an epidemic at home and around the world . Indian J Ophthalmol. 2016, 64:69-75. 10.4103/0301-4738.178150

3. Madsen-Bouterse SA, Kowluru RA: Oxidative stress and diabetic retinopathy: pathophysiological mechanisms and treatment perspectives. Rev Endocr Metab Disord. 2008, 9:315-327. 10.1152/ajpcell.2001.280.4.C719

4. Lum H, Roebuck KA: Oxidant stress and endothelial cell dysfunction. Am J Physiol Cell Physiol. 2001, 280:719-741. 10.1152/ajpcell.2001.280.4.C719

5. Kowluru RA, Chan PS: Oxidative stress and diabetic retinopathy. Exp Diabetes Res. 2007, 2007:43603. $10.1155 / 2007 / 43603$

6. Roeser HP, Lee GR, Nacht S, Cartwright GE: The role of ceruloplasmin in iron metabolism . J Clin Invest. 1970, 49:2408-2417. 10.1172/JCI106460

7. Memişoğullari R, Bakan E: Levels of ceruloplasmin, transferrin, and lipid peroxidation in the serum of patients with Type 2 diabetes mellitus. J Diabetes Complications. 2004, 18:193-197. 10.1016/S1056872700032-1

8. Samokyszyn VM, Miller DM, Reif DW, Aust SD: Inhibition of superoxide and ferritin-dependent lipid peroxidation by ceruloplasmin. J Biol Chem. 1989, 264:21-26. 10.1016/S0021-9258(17)31218-8

9. Daimon M, Susa S, Yamatani K, et al.: Hyperglycemia is a factor for an increase in serum ceruloplasmin in type 2 diabetes. Diabetes Care. 1998, 21:1525-1528. 10.2337/diacare.21.9.1525

10. Inoue K, Sakano N, Ogino K, et al.: Relationship between ceruloplasmin and oxidative biomarkers including ferritin among healthy Japanese. J Clin Biochem Nutr. 2013, 52:160-166. 10.3164/jcbn.12-122

11. Sharma VK, Tumbapo A, Pant V, et al.: Ceruloplasmin, a potential marker for glycemic status and its relationship with lipid profile in Type II diabetes mellitus. Asian J Med Sci. 2018, 9:13-18. 10.3126/ajms.v9i2.19003

12. Du Y, Veenstra A, Palczewski K, Kern TS: Photoreceptor cells are major contributors to diabetes-induced oxidative stress and local inflammation in the retina. Proc Natl Acad Sci U S A. 2013, 110:16586-16591. 10.1073/pnas.1314575110

13. Mohora M, Vîrgolici B, Coman A, Muscurel C, Găman L, Gruia V, Greabu M: Diabetic foot patients with and without retinopathy and plasma oxidative stress. Rom J Intern Med. 2007, 45:51-57.

14. Nowak M, Wielkoszyński T, Marek B, et al.: Antioxidant potential, paraoxonase 1, ceruloplasmin activity and C-reactive protein concentration in diabetic retinopathy. Clin Exp Med. 2010, 10:185-192. 10.1007/s10238009-0084-7

15. Lee MJ, Jung CH, Kang YM, Jang JE, Leem J, Park JY, Lee WJ: Serum ceruloplasmin level as a predictor for the progression of diabetic nephropathy in Korean men with type 2 diabetes mellitus. Diabetes Metab J. 2015, 39:230-239. 10.4093/dmj.2015.39.3.230

16. Zhang R, Li Y, Zhang S, Cai X, Zhou X, Ji L: The association of retinopathy and plasma glucose and HbA1c: a validation of diabetes diagnostic criteria in a Chinese population. J Diabetes Res. 2016, 2016:4034129. $10.1155 / 2016 / 4034129$

17. Bloomgarden Z, Handelsman Y: How does CKD affect HbA1c? . J Diabetes. 2018, 10:270. 10.1111/1753 0407.12624

18. Testa R, Bonfigli AR, Prattichizzo F, La Sala L, De Nigris V, Ceriello A: The "metabolic memory" theory and the early treatment of hyperglycemia in prevention of diabetic complications. Nutrients. 2017, 9:437. 10.3390/nu9050437

19. Dorf A, Ballintine EJ, Bennett PH, Miller M: Retinopathy in Pima Indians. Relationships to glucose level, duration of diabetes, age at diagnosis of diabetes, and age at examination in a population with a high prevalence of diabetes mellitus. Diabetes. 1976, 25:554-560. 10.2337/diab.25.7.554 